\title{
Distributed Finite-Time Consensus Control for Heterogeneous Battery Energy Storage Systems in Droop- Controlled Microgrids
}

DOI:

10.1109/TSG.2018.2868112

\section{Document Version}

Accepted author manuscript

Link to publication record in Manchester Research Explorer

Citation for published version (APA):

Hu, J., \& Lanzon, A. (2019). Distributed Finite-Time Consensus Control for Heterogeneous Battery Energy Storage Systems in Droop-Controlled Microgrids. IEEE Transactions on Smart Grid.

https://doi.org/10.1109/TSG.2018.2868112

\section{Published in:}

IEEE Transactions on Smart Grid

\section{Citing this paper}

Please note that where the full-text provided on Manchester Research Explorer is the Author Accepted Manuscript or Proof version this may differ from the final Published version. If citing, it is advised that you check and use the publisher's definitive version.

\section{General rights}

Copyright and moral rights for the publications made accessible in the Research Explorer are retained by the authors and/or other copyright owners and it is a condition of accessing publications that users recognise and abide by the legal requirements associated with these rights.

\section{Takedown policy}

If you believe that this document breaches copyright please refer to the University of Manchester's Takedown Procedures [http://man.ac.uk/04Y6Bo] or contact uml.scholarlycommunications@manchester.ac.uk providing relevant details, so we can investigate your claim.

\section{OPEN ACCESS}




\title{
Distributed Finite-Time Consensus Control for Heterogeneous Battery Energy Storage Systems in Droop-Controlled Microgrids
}

\author{
Junyan Hu, Student Member, IEEE, and Alexander Lanzon, Senior Member, IEEE
}

\begin{abstract}
This paper presents a novel distributed finite-time control scheme for heterogeneous battery energy storage systems (BESSs) in droop-controlled microgrids. In contrast to the existing centralized methods, the proposed control strategy is fully distributed so that each BESS only requires its own information and the information from its neighbors through a sparse communication network. Our novel consensus-based method is implemented to achieve energy level balancing, active/reactive power sharing, and voltage/frequency synchronization of energy storage devices by using inter-BESS communications, where both the heterogeneous nature of batteries and the hierarchical control structure are taken into consideration. Furthermore, the proposed design is shown to improve the synchronization performance and exhibit more accurate robustness against timevarying communication topologies and load changes. Simulation results on a modified IEEE 57-bus power system are provided to verify the effectiveness of the proposed consensus strategy.
\end{abstract}

Index Terms-Battery energy storage systems, distributed cooperative control, finite-time consensus, microgrid, multi-agent systems

\section{INTRODUCTION}

$\mathbf{M}$ ICROGRIDS are small-scale power systems that facilitate the effective integration of distributed generators, and consist of generation sources, energy storage systems and loads [1]. Since they can be coordinated to achieve autonomous operation, microgrids can be controlled as dispatchable sources to operate in both grid-connected and islanded operating modes [2]. In recent years, battery energy storage systems (BESSs) have been commonly implemented in microgrids in order to increase power quality and network reliability [3]-[5]. Besides this, they can also work as energy buffers to balance renewable supply due to the uncertain behavior of renewable energy sources (i.e. Photovolatic and wind farms).

Conventional control methods for energy storage devices include a centralized control structure which requires a complex, bidirectional, and fully connected communication network [6]. This central controller may suffer from a single point-offailure and adversely affect the reliability, scalability, and flexibility of the whole microgrid system [7], [8]. Alternatively, a distributed cooperative control structure enables each BESS to communicate only with its neighbors through a sparse

This work was supported by the Engineering and Physical Sciences Research Council (EPSRC) [grant number EP/R008876/1]. All research data supporting this publication are directly available within this publication.

The authors are with the School of Electrical and Electronic Engineering, University of Manchester, Manchester, M13 9PL, UK. (e-mail: Junyan.Hu@manchester.ac.uk, Alexander.Lanzon@manchester.ac.uk) communication topology, thus providing a robust secondary control framework that appropriately operates in the presence of time-varying and unreliable communication networks [9][11]. In practice, advanced metering infrastructure (AMI) can be implemented in the microgrids to provide reliable twoway communications between energy storage devices, which lays the foundation of applying distributed cooperative control methods in real applications [12].

Recently, consensus control methods of energy storage systems in microgrids have been investigated in [13]-[16]. For example, an optimal distributed consensus controller for BESSs is proposed in [13]. However, it is necessary to calculate the minimum positive eigenvalue of the communication graph when designing the controller, which means the control strategy is not fully distributed. A distributed cooperative control strategy for state-of-charge (SoC) balancing between the battery modules of a reconfiguration BESS is analyzed in [14], which synchronizes the SoC levels of all battery storage systems in a microgrid using its neighbors' information.

All the mentioned references only consider energy storage systems as homogeneous units and ignore the effect of the heterogeneous nature of batteries. Cooperative control of heterogeneous storage devices in a DC microgrid is proposed in [17], where hybrid energy storage systems consisting of batteries and ultracapacitors are coordinated to achieve SoC balancing and voltage synchronization. However, the effects of droop-based active/reactive power and the frequency of energy storage systems are not considered in the controller design. A multi-agent consensus design for heterogeneous energy storage devices that consider hierarchical control structure is developed in [18], but the proposed asymptotic controller can only guarantee global asymptotic stability of the energy storage devices, meaning that consensus can only be reached over an infinite settling period.

Motivated by the challenges stated above, a distributed finite-time control design for heterogeneous BESSs in microgrids that considers hierarchical control structure with droop characteristics is proposed. The microgrid is viewed as a multi-agent system in which BESSs serve as agents and each BESS communicates with the others through a sparse communication network. Without requiring a central controller, the proposed distributed finite-time controller in this paper is applied to BESSs in order to achieve energy level balancing, active/reactive power sharing, and voltage/frequency restoration within a finite time. Therefore, the contributions in the study can be summarized as follows: 
1) A distributed finite-time control protocol for heterogeneous BESSs that achieves energy level balancing, active/reactive power sharing, and voltage/frequency restoration simultaneously is proposed based on a hierarchical control structure, which could not be achieved in the previous research.

2) The proposed distributed cooperative control method is implemented to provide a reliable hierarchical control structure, where each BESS only uses local information and information from neighboring BESSs through a sparse communication network. Thus, the controller is fully distributed regardless of global information of communication graphs, which largely reduces computational and communication burdens comparing with centralized methods.

3) In comparison with conventional distributed controllers, the proposed method shows more robustness against load changes and time-varying communication topologies. Besides, it yields better disturbance rejection properties and a faster convergence speed when compared with other existing distributed methods.

The paper is organized as follows. Preliminaries on graph theory and the cooperative control problem of BESSs are formulated in Section II. Distributed finite-time controller design for BESSs is presented in Section III. The proposed control protocols are validated by simulation case studies in Section IV. Conclusions are given in Section V.

Throughput this paper, let $I_{n} \in \mathbb{R}^{n \times n}$ denote the identity matrix of dimension $n$ and $\mathbf{1}_{n} \in \mathbb{R}^{n}$ be the vector with all entries equal to one. $\operatorname{diag}\left\{a_{i}\right\}$ represents a diagonal matrix with diagonal entries $a_{i} \cdot \operatorname{sgn}($.$) is the signum function.$

\section{PRoblem Formulation}

\section{A. Preliminaries on Graph Theory}

The microgrid is usually recognized as a multi-agent cooperative system, in which BESSs and communication lines play the roles of agents and edges. Each BESS can communicate with other BESSs through an undirected communication network. Consider a weighted and undirected graph $\mathcal{G}=$ $(\mathcal{V}, \mathcal{E}, \mathcal{A})$ with a nonempty set of $N$ nodes $\mathcal{V} \in\{1,2, \ldots, N\}$, a set of edges $\mathcal{E} \subset \mathcal{V} \times \mathcal{V}$, and associated adjacency matrix $\mathcal{A}=\left[a_{i j}\right]_{N \times N}$. An edge rooted at node $i$ and ended at node $j$ is denoted by $(i, j)$, which means information can flow from node $i$ to node $j . a_{i j}$ is the weight of edge $(i, j)$ and $a_{i j}=a_{j i}>0$ if $(i, j) \in \mathcal{E}$. Assume that there are no repeated edges and no self loops. Node $j$ is called a neighbour of node $i$ if $(i, j) \in \mathcal{E}$. Define the in-degree matrix as $D=\operatorname{diag}\left\{d_{i}\right\} \in \mathbb{R}^{N \times N}$ with $d_{i}=\sum_{j=1}^{N} a_{i j}$. The Laplacian matrix $L \in \mathbb{R}^{N \times N}$ of $\mathcal{G}$ is defined as $L=D-\mathcal{A}$. If node $i$ observes the leader, an edge $(0, i)$ is said to exist with weighting gain $g_{i}>0$ as a pinned node. We denote the pinning matrix as $G=\operatorname{diag}\left\{g_{i}\right\} \in \mathbb{R}^{N \times N}$.

\section{B. Battery Energy Storage System}

Assume there are $N$ BESSs connected by a sparse undirected communication graph in an autonomous microgrid. The structure of a Li-ion BESS in the microgrid is depicted in

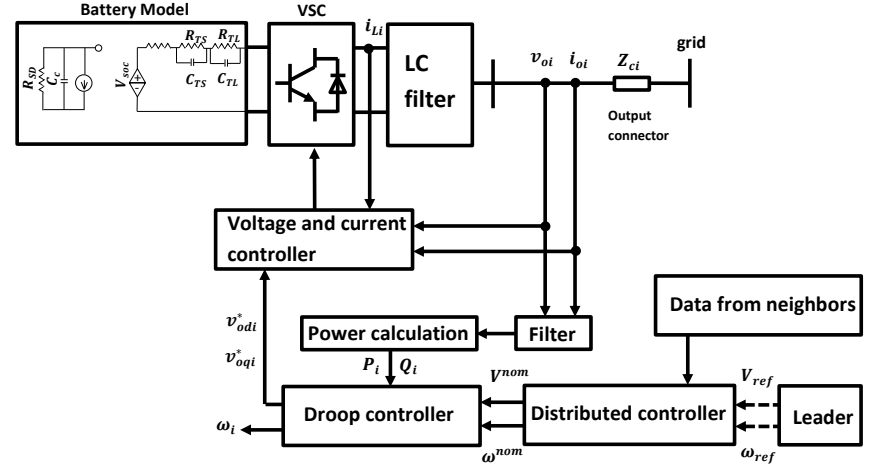

Fig. 1. Block diagram of battery energy storage systems

Fig. 1, where each energy storage system contains a battery energy source, a DC/AC inverter bridge, a grid filter, and hierarchical control loops. Vector control is implemented in order to convert the rotating $a b c$ frame to the $d q$ frame. As illustrated in Fig. 1, the hierarchical control of energy storage devices has three main control loops, namely, fast inner voltage and current controller, droop controller, and distributed controller. Because of the use of droop techniques in primary control, the distributed controller will synchronize energy levels, active/reactive power sharing, voltage magnitude, and frequency by providing the nominal voltage and frequency references to the droop controller.

In the power calculation block, the measured active and reactive powers can be derived as

$$
\begin{aligned}
& \dot{P}_{i}=-\omega_{c i} P_{i}+\frac{3}{2} \omega_{c i}\left(v_{\text {odi }} i_{\text {od } i}+v_{\text {oq } i} i_{\text {oq } i}\right), \\
& \dot{Q}_{i}=-\omega_{c i} Q_{i}+\frac{3}{2} \omega_{c i}\left(v_{\text {odi }} i_{\text {oq } i}-v_{\text {oqi }} i_{\text {odi }}\right),
\end{aligned}
$$

where $v_{\text {odi }}, v_{\text {oq } i}, i_{\text {odi }}$, and $i_{\text {oqi }}$ are the direct and quadrature components of $v_{o i}$ and $i_{o i}$ in Fig. $1, \omega_{c i}$ is the cutoff frequency of the low-pass filters used in measuring power, and $P_{i}$ and $Q_{i}$ are measured active and reactive powers at terminals of the $i$ th BESS inverter, respectively.

When BESSs are connected in parallel, the droop controller is in charge of controlling the voltage magnitude and the frequency using locally measured active and reactive powers. The voltage and frequency droop characteristics of the $i$ th battery are presented by the following equations:

$$
\begin{aligned}
\omega_{i} & =\omega_{i}^{n o m}-K_{i}^{P} P_{i}, \\
v_{o d i}^{*} & =V_{i}^{n o m}-K_{i}^{Q} Q_{i}, \\
v_{o q i}^{*} & =0,
\end{aligned}
$$

where $K_{i}^{P}$ and $K_{i}^{Q}$ are droop coefficients selected based on the inverter's active and reactive power ratings, $v_{\text {odi }}^{*}$ and $v_{\text {oq } i}^{*}$ are the reference voltage signals of the $i$ th energy storage, and $V_{i}^{\text {nom }}$ and $\omega_{i}^{\text {nom }}$ are the set points of the primary control [7].

Since the dynamics of the voltage and current control loops are much faster than the dynamics of droop control loop [2], they are neglected in the modeling for brevity. According to $d-q$ transmission, the output voltage magnitude of BESS unit is given by

$$
V_{i}=\sqrt{v_{o d i}^{2}+v_{o q i}^{2}}
$$


thus we can replace $v_{o d i}^{*}$ with $V_{i}$ in (2b) to represent the dynamics of voltage magnitude in the $i^{\text {th }}$ BESS.

Based on the soc-based battery model developed in [19], the simplified battery dynamics shown in [20] are selected for large-scale power systems with multiple energy storage devices. A similar model is also employed in [21], [22]. However, the battery model in [20]-[22] ignores the effect of droop control and assumes that all batteries are homogeneous. In this paper, the design method takes the dynamics of droop controllers and the heterogeneous nature of each battery into consideration so that the BESS model can be formulated as follows:

$$
\begin{aligned}
\dot{E}_{i} & =\frac{-K_{i}^{E}}{3600} P_{i}+u_{i}^{E}, \\
\dot{P}_{i} & =u_{i}^{P}, \\
V_{i} & =V_{i}^{n o m}-K_{i}^{Q} Q_{i}, \\
\omega_{i} & =\omega_{i}^{n o m}-K_{i}^{P} P_{i},
\end{aligned}
$$

where coefficient $K_{i}^{E}$ represents the heterogeneous nature of each battery in the droop-controlled microgrid, and $u_{i}^{E}$ and $u_{i}^{P}$ are control inputs of energy level and active power of the $i^{\text {th }}$ BESS, respectively. It is shown that the energy level and power of each BESS are controlled independently in our paper in order to lead a fast consensus, whereas other existing research [14], [20] show only one control input $u_{i}^{P}$ for controlling both energy levels and the active powers.

Consider that the voltage magnitude and frequency are controlled by regulating nominal reference points $V_{i}^{\text {nom }}$ and $\omega_{i}^{\text {nom }}$ respectively, new control inputs can be designed to synchronize voltage magnitude and frequency of BESSs by regulating $V_{i}^{\text {nom }}$ and $\omega_{i}^{\text {nom }}$. Both local and neighbors' information of active and reactive power will be fed into the integral control installed at each battery converter. Finally, considering the hierarchical control, the dynamic model of the $i^{\text {th }}$ BESS can be given by

$$
\begin{aligned}
\dot{E}_{i} & =\frac{-K_{i}^{E}}{3600} P_{i}+u_{i}^{E}, \\
\dot{P}_{i} & =u_{i}^{P}, \\
\dot{Q}_{i} & =u_{i}^{Q}, \\
V_{i} & =V_{i}^{n o m}-K_{i}^{Q} Q_{i}, \\
\dot{V}_{i}^{n o m} & =u_{i}^{V}+K_{i}^{Q} u_{i}^{Q}, \\
\omega_{i} & =\omega_{i}^{n o m}-K_{i}^{P} P_{i}, \\
\dot{\omega}_{i}^{n o m} & =u_{i}^{\omega}+K_{i}^{P} u_{i}^{P},
\end{aligned}
$$

where $u_{i}^{Q}, u_{i}^{V}$, and $u_{i}^{\omega}$ are the control inputs of reactive power, voltage magnitude, and frequency of the $i^{\text {th }}$ BESS, respectively.

The control objective is to design suitable distributed cooperative controllers such that the voltage magnitudes and frequencies of networked BESSs are restored to the desired nominal values within a finite time period. Furthermore, the synchronizations of energy levels and active/reactive power sharing among storage devices also need to be guaranteed.

\section{Distributed Finite-Time Consensus CONTROL DESIGN FOR BESSS}

In this section, we will design a distributed finite-time consensus control scheme to synchronize battery energy levels and active/reactive power sharing while voltage magnitudes and frequencies of each BESS track their reference values.

\section{A. Distributed Finite-Time Control for Battery Energy and Active/Reactive Powers}

In the droop-controlled microgrids, it is expected that energy storage devices with low initial energy levels will run out of energy first in the discharging mode while those with high initial energy levels may be overloaded in the charging mode and thus waste the generation potential of renewable energy sources. Therefore, BESSs can only contribute their full power capacity to deal with generation and demand fluctuations when all the energy devices approach a common energy level [13]. In the heterogeneous BESSs, the different efficiency and different cost of use can be normalized to a common scale such that it is still practical to maintain an equal level for all the BESSs in the microgrid. Toward this end, a distributed finite-time control structure utilizing neighbor to neighbor communication is developed to achieve a balanced energy level among the energy storage devices while allowing the BESSs to make full use of their power capacities.

Note that the active/reactive power sharing among each BESS is provided by primary control according to

$$
\begin{gathered}
\frac{P_{j}}{P_{i}}=\frac{K_{i}^{P}}{K_{j}^{P}}, \quad \forall i, j \in\{1, \ldots, N\}, \\
\frac{Q_{j}}{Q_{i}}=\frac{K_{i}^{Q}}{K_{j}^{Q}}, \quad \forall i, j \in\{1, \ldots, N\} .
\end{gathered}
$$

Denote $\tilde{P}_{i}=K_{i}^{P} P_{i}$ and $\tilde{Q}_{i}=K_{i}^{Q} Q_{i}, \forall i \in\{1, \ldots, N\}$. The active/reactive sharing accuracy of BESSs can be guaranteed by the consensus of $\tilde{P}_{i}$ and $\tilde{Q}_{i}$. Motivated by [23], we propose the following distributed finite-time protocols

$$
\begin{gathered}
u_{i}^{E}=c_{E} \sum_{j=1}^{N} a_{i j} \operatorname{sgn}\left(E_{j}-E_{i}\right)\left|E_{j}-E_{i}\right|^{\alpha}, \\
u_{i}^{P}=\frac{c_{P}}{K_{i}^{P}} \sum_{j=1}^{N} a_{i j} \operatorname{sgn}\left(\tilde{P}_{j}-\tilde{P}_{i}\right)\left|\tilde{P}_{j}-\tilde{P}_{i}\right|^{\alpha}, \\
u_{i}^{Q}=\frac{c_{Q}}{K_{i}^{Q}} \sum_{j=1}^{N} a_{i j} \operatorname{sgn}\left(\tilde{Q}_{j}-\tilde{Q}_{i}\right)\left|\tilde{Q}_{j}-\tilde{Q}_{i}\right|^{\alpha},
\end{gathered}
$$

where $c_{E}, c_{P}$, and $c_{Q}$ are positive control gains, and $0<\alpha<$ 1 .

Define the consensus error of the active and reactive powers as

$$
\begin{aligned}
& \xi_{P i}(t)=\tilde{P}_{i}(t)-\frac{1}{N} \sum_{i=1}^{N} \tilde{P}_{i}(t), \\
& \xi_{Q i}(t)=\tilde{Q}_{i}(t)-\frac{1}{N} \sum_{i=1}^{N} \tilde{Q}_{i}(t) .
\end{aligned}
$$


For an undirected and connected communication graph $\mathcal{G}$, we have $\frac{1}{N} \sum_{i=1}^{N} \dot{\tilde{P}}_{i}(t)=\frac{1}{N} \sum_{i=1}^{N} \dot{\tilde{Q}}_{i}(t)=0$, which means $\frac{1}{N} \sum_{i=1}^{N} \tilde{P}_{i}(t)$ and $\frac{1}{N} \sum_{i=1}^{N} \tilde{Q}_{i}(t)$ are time invariant.

Then we have the differential of error systems as follows

$$
\begin{aligned}
\dot{\xi}_{P i}(t) & =\dot{\tilde{P}}_{i}(t)-\frac{1}{N} \sum_{i=1}^{N} \dot{\tilde{P}}_{i}(t)=K_{i}^{P} u_{i}^{P} \\
& =c_{P} \sum_{j=1}^{N} a_{i j} \operatorname{sgn}\left(\tilde{P}_{j}-\tilde{P}_{i}\right)\left|\tilde{P}_{j}-\tilde{P}_{i}\right|^{\alpha} \\
& =c_{P} \sum_{j=1}^{N} a_{i j} \operatorname{sgn}\left(\xi_{P j}-\xi_{P i}\right)\left|\xi_{P j}-\xi_{P i}\right|^{\alpha} \\
\dot{\xi}_{Q i}(t) & =\dot{\tilde{Q}}_{i}(t)-\frac{1}{N} \sum_{i=1}^{N} \dot{\tilde{Q}}_{i}(t)=K_{i}^{Q} u_{i}^{Q} \\
& =c_{Q} \sum_{j=1}^{N} a_{i j} \operatorname{sgn}\left(\tilde{Q}_{j}-\tilde{Q}_{i}\right)\left|\tilde{Q}_{j}-\tilde{Q}_{i}\right|^{\alpha} \\
& =c_{Q} \sum_{j=1}^{N} a_{i j} \operatorname{sgn}\left(\xi_{Q j}-\xi_{Q i}\right)\left|\xi_{Q j}-\xi_{Q i}\right|^{\alpha}
\end{aligned}
$$

Lemma 1 ( [24]): If $a_{1}, a_{2}, \ldots, a_{n} \geq 0$, then for $0<r<p$

$$
\left(\sum_{i=1}^{n} a_{i}^{p}\right)^{1 / p} \leq\left(\sum_{i=1}^{n} a_{i}^{r}\right)^{1 / r} .
$$

Lemma 2 ( [25]): For an undirected graph $\mathcal{G}$, the Laplacian matrix $L$ has the following properties.

1) $x^{T} L x=\frac{1}{2} \sum_{i, j=1}^{N} a_{i j}\left(x_{j}-x_{i}\right)^{2}$, and $L$ is positive semidefinite.

2) Let $\lambda_{2}(L)$ be the second smallest eigenvalue of $L$. If $\mathbf{1}^{T} x=$ 0 , one has $x^{T} L x \geq \lambda_{2}(L) x^{T} x$.

Lemma 3 ( [26]): Suppose that function $V(x): \mathbb{R}^{n} \rightarrow \mathbb{R}$ is C-regular, and that $x(t):[0,+\infty) \rightarrow \mathbb{R}^{n}$ is absolutely continuous on any compact interval of $[0,+\infty)$. If there exists $K>0$ and $0<\alpha<1$, such that

$$
\frac{d V(t)}{d t} \leq-K V^{\alpha}(t)
$$

then $V(t)=0$ for all $t \geq t^{*}$, and the settling time $t^{*}$ can be estimated by

$$
t^{*}=\frac{V^{1-\alpha}(0)}{K(1-\alpha)} .
$$

Theorem 1: Let the undirected communication graph $\mathcal{G}$ be connected, by using the distributed finite-time control protocols (8)-(10), the consensus of energy levels and active/reactive power sharing accuracy of all BESSs are guaranteed.

Proof: It can be seen that (13) and (14) have the same form, so only the consensus proof for active power is considered here and the consensus proof for reactive power can be derived similarly.

Define an undirected graph $\mathcal{G}_{P}$ with adjacency matrix $\mathcal{A}_{P}=$ $\left[a_{P i j}\right]_{N \times N}=\left[\left(c_{P} a_{i j}\right)^{\frac{2}{1+\alpha}}\right]_{N \times N}$ and Laplacian matrix $L_{P}$. Denote $\lambda_{2}$ the smallest positive eigenvalue of $L_{P}$.
Consider the following Lyapunov function candidate

$$
V_{1}=2 \lambda_{2} \xi^{T}(t) \xi(t)=2 \lambda_{2} \sum_{i=1}^{N} \xi_{P i}^{2}(t)
$$

where $\xi(t)=\left[\xi_{P 1}(t), \xi_{P 2}(t), \ldots, \xi_{P N}(t)\right]^{T}$ is the disagreement vector.

Thus, the time derivative of $V_{1}$ is obtained as

$$
\begin{aligned}
\dot{V}_{1}= & 4 \lambda_{2} \sum_{i=1}^{N} \xi_{P i}(t) \dot{\xi}_{P i}(t) \\
= & 4 \lambda_{2} \sum_{i=1}^{N} \xi_{P i}(t) \sum_{j=1}^{N} a_{P i j}^{\frac{1+\alpha}{2}} \operatorname{sgn}\left(\xi_{P j}-\xi_{P i}\right)\left|\xi_{P j}-\xi_{P i}\right|^{\alpha} \\
= & 2 \lambda_{2} \sum_{i, j=1}^{N} a_{P i j}^{\frac{1+\alpha}{2}} \xi_{P i} \operatorname{sgn}\left(\xi_{P j}-\xi_{P i}\right)\left|\xi_{P j}-\xi_{P i}\right|^{\alpha} \\
& +2 \lambda_{2} \sum_{i, j=1}^{N} a_{P j i}^{\frac{1+\alpha}{2}} \xi_{P j} \operatorname{sgn}\left(\xi_{P i}-\xi_{P j}\right)\left|\xi_{P i}-\xi_{P j}\right|^{\alpha} \\
= & 2 \lambda_{2} \sum_{i, j=1}^{N} a_{P i j}^{\frac{1+\alpha}{2}}\left(\xi_{P i}-\xi_{P j}\right) \operatorname{sgn}\left(\xi_{P j}-\xi_{P i}\right)\left|\xi_{P j}-\xi_{P i}\right|^{\alpha} \\
= & -2 \lambda_{2} \sum_{i, j=1}^{N} a_{P i j}^{\frac{1+\alpha}{2}}\left|\xi_{P j}-\xi_{P i}\right|^{1+\alpha}
\end{aligned}
$$

By using Lemma 1 , for $0<\alpha<1$, we can obtain

$$
\begin{gathered}
\left(\sum_{i, j=1}^{N} a_{P i j}^{\frac{1+\alpha}{2}}\left|\xi_{P j}-\xi_{P i}\right|^{1+\alpha}\right)^{\frac{1}{1+\alpha}} \\
\leq\left(\sum_{i, j=1}^{N} a_{P i j}\left|\xi_{P j}-\xi_{P i}\right|^{2}\right)^{\frac{1}{2}}
\end{gathered}
$$

Then it follows from Lemma 2 that

$$
\begin{aligned}
\dot{V}_{1} & \leq-2 \lambda_{2}\left(\sum_{i, j=1}^{N} a_{P i j}\left|\xi_{P j}-\xi_{P i}\right|^{2}\right)^{\frac{1+\alpha}{2}} \\
& =-2 \lambda_{2}\left[2 \xi^{T}(t) L_{P} \xi(t)\right]^{\frac{1+\alpha}{2}} \\
& \leq-2 \lambda_{2}\left[2 \lambda_{2} \xi^{T}(t) \xi(t)\right]^{\frac{1+\alpha}{2}} \\
& =-2 \lambda_{2}\left[V_{1}(t)\right]^{\frac{1+\alpha}{2}}
\end{aligned}
$$

According to Lemma 3, the finite-time average-consensus active power sharing problem is solved by control protocol (9) with $0<\alpha<1$. The convergence time can be upper bounded in terms of the initial errors as

$$
T_{\sigma}(\alpha)=\frac{V_{1}^{\frac{1-\alpha}{2}}(0)}{2 \lambda_{2} \frac{1-\alpha}{2}}=\frac{\left[2 \lambda_{2}\|\xi(0)\|^{2}\right]^{\frac{1-\alpha}{2}}}{(1-\alpha) \lambda_{2}} .
$$

As a result, the finite-time average consensus of active power sharing in energy storage devices is achieved, such that

$$
\left\{\begin{array}{l}
\lim _{t \rightarrow T_{\sigma}} \tilde{P}_{i}(t)=\tilde{P}_{j}(t) \\
\tilde{P}_{i}(t)=\tilde{P}_{j}(t), \quad \forall t \geq T_{\sigma}
\end{array}\right.
$$


for all $i, j \in\{1, \ldots, N\}$. Same analysis holds for the finitetime consensus of ractive power sharing of energy storage systems.

Next, we will show that BESS's energy levels reach consensus within finite-time under protocol (8).

Let $K_{i}^{P}=K_{i}^{E}>0$, the transients of energy levels can be expressed by

$$
E_{i}(t)=\hat{E}_{i}(t)-\frac{1}{3600} \int_{0}^{t} \tilde{P}_{i}(\tau) d \tau
$$

where $\hat{E}_{i}(t)$ is the solution of the equation $\dot{E}_{i}(t)=u_{i}^{E}$. It can be seen that the dynamics of $\hat{E}_{i}$ is also in form of a consensus controller for single integrator agents, by using a similar proof as above, we can prove that $\hat{E}_{i}(t)$ consensus to $\frac{1}{N} \sum_{i=1}^{N} E_{i}(t)$ in finite time $T_{E}(\alpha)$. Consider that the active power achieves average-consensus within finite time $T_{\sigma}(\alpha)$, the energy levels of all BESSs satisfy

$$
\left\{\begin{array}{l}
\lim _{t \rightarrow T_{E}} E_{i}(t)=\frac{1}{N} \sum_{i=1}^{N} E_{i}(t)-\frac{1}{3600 N} \sum_{i=1}^{N} \tilde{P}_{i}(t) t \\
E_{i}(t)=\frac{1}{N} \sum_{i=1}^{N} E_{i}(t)-\frac{1}{3600 N} \sum_{i=1}^{N} \tilde{P}_{i}(t) t, \quad \forall t \geq T_{E},
\end{array}\right.
$$

which indicates that all energy levels reaches consensus within finite time. This completes the proof.

Remark 1: It is worth mentioning that $1 / K_{i}^{P}$ is the active power ratio of the $i^{\text {th }}$ BESS, which also reflects the heterogeneous nature of the batteries, such that the selection of $K_{i}^{P}=K_{i}^{E} \forall i \in\{1, \ldots, N\}$ is reasonable.

Remark 2: The proposed distributed cooperative control in this paper is different from that in [13], where it is necessary to calculate the minimum nonzero eigenvalue of the global communication topology when designing the controller. Our distributed finite-time controller allows each BESS only to access the information from its neighbors such that the communication costs are greatly reduced and the proposed method is fully distributed. Besides this, the control design in [18] is a asymptotic controller which can only guarantee global asymptotic stability of the energy storage system, whereas with our proposed method, the synchronization of energy levels and active/reactive powers is achieved within a finite time period.

Remark 3: Due to the intermittency of renewable generation such as photovoltaic and wind power, BESSs may be installed in a hybrid microgrid to provide a faster response to absorb excessive power and compensate the insufficient power during peak generation and load periods. Since the proposed BESS model is developed based on the relationship between measured energy level and power, the impact of other distributed energy resources can be viewed as model uncertainties and the performance of the BESSs can be guaranteed by the robustness of the designed controller [27], [28]. Furthermore, the proposed control architecture can also be slightly modified to achieve better performance in a hybrid microgrid by taking accurate energy resource models into consideration as analyzed in [17], [29]-[31].

\section{B. Distributed Finite-Time Control for Voltage Magnitude and Frequency}

In this section, a novel distributed finite-time cooperative tracking strategy is proposed to synchronize the BESS voltage magnitude $V_{i}$ and frequecy $\omega_{i}$ to the nominal voltage reference $V_{\text {ref }}$ and frequency reference $\omega_{\text {ref }}$, respectively.

Assume that each BESS only requires its own information and the information from its neighboring BESSs over an undirected communication network, and at least one BESS can observe the reference. Similar to the previous section, the distributed finite-time voltage and frequency protocols are designed as follows

$$
\begin{aligned}
u_{i}^{V}= & c_{V}\left[\sum_{j=1}^{N} a_{i j} \operatorname{sgn}\left(V_{j}-V_{i}\right)\left|V_{j}-V_{i}\right|^{\alpha}\right. \\
& \left.+g_{i} \operatorname{sgn}\left(V_{\text {ref }}-V_{i}\right)\left|V_{\text {ref }}-V_{i}\right|^{\alpha}\right], \\
u_{i}^{\omega}= & c_{\omega}\left[\sum_{j=1}^{N} a_{i j} \operatorname{sgn}\left(\omega_{j}-\omega_{i}\right)\left|\omega_{j}-\omega_{i}\right|^{\alpha}\right. \\
& \left.+g_{i} \operatorname{sgn}\left(\omega_{\text {ref }}-\omega_{i}\right)\left|\omega_{\text {ref }}-\omega_{i}\right|^{\alpha}\right] .
\end{aligned}
$$

where $c_{V}$ and $c_{\omega}$ are positive constant gains.

Next, we define the consensus tracking error of voltage magnitude and frequency as

$$
\xi_{V i}(t)=V_{i}(t)-V_{r e f}
$$

and

$$
\xi_{\omega i}(t)=\omega_{i}(t)-\omega_{r e f} .
$$

Differentiating the error terms yields

$$
\begin{aligned}
\dot{\xi}_{V i}= & \dot{V}_{i}-\dot{V}_{\text {ref }}=u_{i}^{V} \\
= & c_{V}\left[\sum_{j=1}^{N} a_{i j} \operatorname{sgn}\left(V_{j}-V_{i}\right)\left|V_{j}-V_{i}\right|^{\alpha}\right. \\
& \left.+g_{i} \operatorname{sgn}\left(V_{r e f}-V_{i}\right)\left|V_{r e f}-V_{i}\right|^{\alpha}\right] \\
= & c_{V}\left[\sum_{j=1}^{N} a_{i j} \operatorname{sgn}\left(\xi_{V j}-\xi_{V i}\right)\left|\xi_{V j}-\xi_{V i}\right|^{\alpha}\right. \\
& \left.-g_{i} \operatorname{sgn}\left(\xi_{V i}\right)\left|\xi_{V i}\right|^{\alpha}\right]
\end{aligned}
$$

and

$$
\begin{aligned}
\dot{\xi}_{\omega i}= & \dot{\omega}_{i}-\dot{\omega}_{r e f}=u_{i}^{\omega} \\
= & c_{\omega}\left[\sum_{j=1}^{N} a_{i j} \operatorname{sgn}\left(\omega_{j}-\omega_{i}\right)\left|\omega_{j}-\omega_{i}\right|^{\alpha}\right. \\
& \left.+g_{i} \operatorname{sgn}\left(\omega_{r e f}-\omega_{i}\right)\left|\omega_{r e f}-\omega_{i}\right|^{\alpha}\right] \\
= & c_{\omega}\left[\sum_{j=1}^{N} a_{i j} \operatorname{sgn}\left(\xi_{\omega j}-\xi_{\omega i}\right)\left|\xi_{\omega j}-\xi_{\omega i}\right|^{\alpha}\right. \\
& \left.-g_{i} \operatorname{sgn}\left(\xi_{\omega i}\right)\left|\xi_{\omega i}\right|^{\alpha}\right] .
\end{aligned}
$$

Lemma 4 ( [6]): For an undirected graph $\mathcal{G}$, the Laplacian matrix $(L+G)$ has the following properties.

1) $x^{T}(L+G) x=\frac{1}{2} \sum_{i, j=1}^{N} a_{i j}\left(x_{j}-x_{i}\right)^{2}+\sum_{i=1}^{N} g_{i} x_{i}^{2}$, and $(L+G)$ is positive definite.

2) Let $\lambda_{1}(L+G)$ be the smallest eigenvalue of $(L+G)$, then one has $x^{T}(L+G) x \geq \lambda_{1}(L+G) x^{T} x$. 
Theorem 2: Let the undirected communication graph $\mathcal{G}$ be connected, by using the distributed finite-time control protocols (23) and (24), the voltage magnitude and frequency of all storage devices converge to their reference values within finite time.

Proof: It is observed that the dynamics of voltage magnitude and frequency have the same form, only consensus tracking of voltage magnitude are provided here for brevity.

Define an undirected graph $\mathcal{G}_{V}$ with $a_{V i j}=\left(c_{V} a_{i j}\right)^{\frac{2}{1+\alpha}}$ and $g_{V i}=\left(c_{V} g_{i}\right)^{\frac{2}{1+\alpha}} . L_{V}$ is the Laplacian matrix of the graph $\mathcal{G}_{V}$ and $G_{V}=\operatorname{diag}\left(g_{V i}\right) \in \mathbb{R}^{N \times N}$ is diagonal matrix of its pinning gains. Denote $\lambda_{1}$ the smallest eigenvalue of $\left(L_{V}+G_{V}\right)$.

Consider the following Lyapunov function candidate

$$
V_{2}=2 \lambda_{1} \zeta^{T}(t) \zeta(t)=2 \lambda_{1} \sum_{i=1}^{N} \xi_{V i}^{2}(t)
$$

where $\zeta(t)=\left[\xi_{V 1}(t), \xi_{V 2}(t), \ldots, \xi_{V N}(t)\right]^{T}$ is the disagreement vector.

Similar to the proof of Theorem 1, the time derivative of $V_{2}$ is obtained as

$$
\begin{aligned}
\dot{V}_{2}= & 4 \lambda_{1} \sum_{i=1}^{N} \xi_{V i}(t) \dot{\xi}_{V i}(t) \\
= & -2 \lambda_{1} \sum_{i, j=1}^{N} a_{V i j}^{\frac{1+\alpha}{2}}\left|\xi_{V j}-\xi_{V i}\right|^{1+\alpha} \\
& -4 \lambda_{1} \sum_{i=1}^{N} g_{V i}^{\frac{1+\alpha}{2}}\left|\xi_{V i}\right|^{1+\alpha} .
\end{aligned}
$$

Based on Lemma 1 and Lemma 4, one has

$$
\begin{aligned}
\dot{V}_{2} & \leq-2 \lambda_{1}\left(\sum_{i, j=1}^{N} a_{V i j}\left|\xi_{V j}-\xi_{V i}\right|^{2}+2 \sum_{i=1}^{N} g_{V i}\left|\xi_{V i}\right|^{2}\right)^{\frac{1+\alpha}{2}} \\
& =-2 \lambda_{1}\left[2 \zeta^{T}(t)\left(L_{V}+G_{V}\right) \zeta(t)\right]^{\frac{1+\alpha}{2}} \\
& \leq-2 \lambda_{1}\left[2 \lambda_{1} \zeta^{T}(t) \zeta(t)\right]^{\frac{1+\alpha}{2}} \\
& =-2 \lambda_{1}\left[V_{2}(t)\right]^{\frac{1+\alpha}{2}}
\end{aligned}
$$

According to Lemma 3, the consensus tracking error reaches 0 at finite time

$$
T_{\eta}(\alpha)=\frac{V_{2}^{\frac{1-\alpha}{2}}(0)}{2 \lambda_{1} \frac{1-\alpha}{2}}=\frac{\left[2 \lambda_{1}\|\zeta(0)\|^{2}\right]^{\frac{1-\alpha}{2}}}{(1-\alpha) \lambda_{1}} .
$$

Therefore, the distributed finite-time tracking of voltage magnitude in BESSs is achieved, such that all $N$ BESSs satisfy

$$
\left\{\begin{array}{l}
\lim _{t \rightarrow T_{\eta}} V_{i}(t)=V_{\text {ref }} \\
V_{i}(t)=V_{\text {ref }}, \forall t \geq T_{\eta}
\end{array}\right.
$$

for all $i \in\{1, \ldots, N\}$. Same analysis holds for the finitetime tracking of frequencies of energy storage systems. This completes the proof.

Remark 4: It should be noted that when $\alpha=0$ the finitetime protocol can still guarantee the consensus of voltage and frequency in a microgrid as shown in [32], but the error system

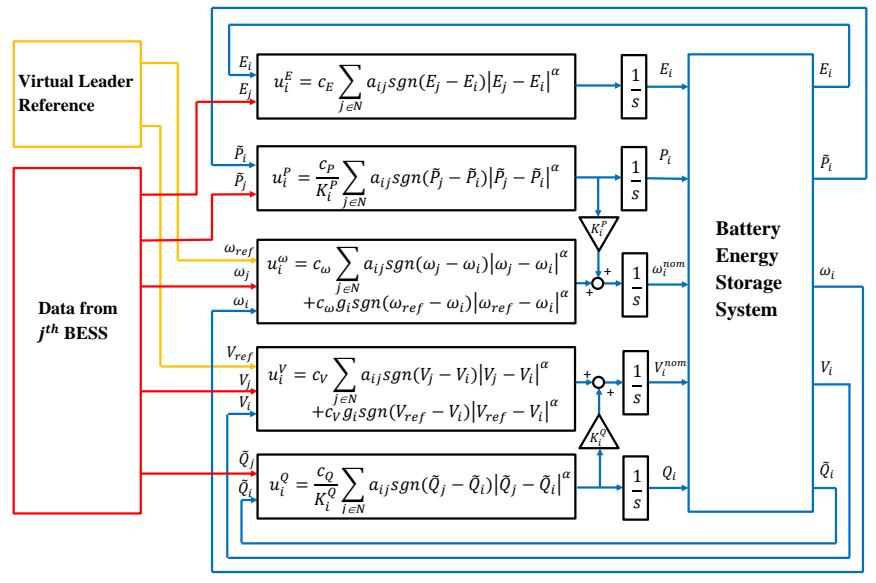

Fig. 2. The diagram of distributed finite-time control scheme of BESSs

becomes discontinuous. Thus, the finite-time method in [32] can be viewed as a special case of the result in the current paper.

Remark 5: One of the conditions for the proposed finite-time consensus control strategy is the connectivity of the communication network. Therefore, when the unexpected faults of BESSs occurred, the performance of the proposed cooperative control algorithm can be guaranteed if the rest of BESSs still remain connected by the communication network. Since the distributed information is used in our controller design instead of global information, the microgrid is also robust to link failures if the sparse communication topology is switched to an appropriate structure in time such that all the BESSs in the microgrid are still connected.

The block diagram of distributed finite-time control scheme for BESSs is shown in Fig. 2, where the consensus of energy storage systems will be achieved autonomously via such a distribution communication manner without global information.

\section{Simulation Results}

In this paper, we have designed a distributed cooperative controller that only exploits local information in a distributed manner such that the battery energy levels are maintained at an equal level for multiple heterogeneous BESSs. As an microgrid may also contain renewable energy sources and various loads, by balancing the energy levels of multiple BESSs at a consensus, it is guaranteed that no battery in the microgird is completely depleted or charged when there exists available energy or free capacity in other BESSs.

To verify the effectiveness of the proposed distributed finitetime control algorithm, a modified IEEE 57-bus system is tested in Matlab/Simulink. The original IEEE 57-bus system has 7 generators, which are now replaced with BESSs as shown in Fig. 3. All the data for the IEEE 57-bus system used in the simulation is represented in [33].

The configuration of the BESS is presented in Fig. 1, where the battery dynamics in [34] is adopted in the energy storage model. The full capacity of the battery $C_{c}$ is given by

$$
C_{c}=3600 C_{n} f_{1} f_{2},
$$


TABLE I

PARAMETERS OF THE BATTERY

\begin{tabular}{ccc}
\hline \hline Symbol & Value & Description \\
\hline$C_{c}$ & $39 e^{5} \mathrm{~F}$ & The battery's capacity \\
$R_{S D}$ & $7.4 e^{-3} \Omega$ & The series resistor \\
$R_{T S}$ & $4.6 e^{-3} \Omega$ & The short transient resistor \\
$C_{T S}$ & $7.036 e^{4} \mathrm{~F}$ & The short transient capacitor \\
$R_{T L}$ & $4.98 e^{-2} \Omega$ & The long transient resistor \\
$C_{T L}$ & $4.47 e^{5} \mathrm{~F}$ & The long transient capacitor \\
\hline \hline
\end{tabular}

where $C_{n}$ is the nominal capacity, and $f_{1}$ and $f_{2}$ are cycle number and temperature-dependent correction factors respectively. Parameters of the battery are functions of state of charge $(S o C)$, which can be expressed by

$$
\begin{aligned}
V_{\text {soc }}= & -1.031 e^{-35 S o C}+3.685+0.2156 S o C \\
& -0.1178 S o C^{2}+0.3201 S o C^{3} \\
R_{S D}= & 0.1562 e^{-24.37 S o C}+0.07446 \\
R_{T S}= & 0.3208 e^{-29.14 S o C}+0.04669 \\
C_{T S}= & -752.9 e^{-13.51 S o C}+703.6 \\
R_{T L}= & 6.603 e^{-155.2 S o C}+0.04984 \\
C_{T L}= & -6065 e^{-27.12 S o C}+4475
\end{aligned}
$$

where the definitions and values of these parameters are illustrated in Table I. The BESSs are connected to the main grid through the Point of Common Coupling (PCC), which is used to measure the power delivered/withdrawn and decide the operation mode of the microgrid [35].

The distributed finite-time consensus protocols are applied to each BESS over undirected communication networks as shown in Fig. 4.

Based on the heterogeneous BESS models developed in Section II, the control parameters of the distributed finitetime protocols are selected as follows: $c_{E}=0.7, c_{P}=0.5$, $c_{Q}=0.45, c_{V}=0.8, c_{\omega}=0.7, K_{1}^{P}=K_{1}^{E}=1.1$, $K_{2}^{P}=K_{2}^{E}=1.3, K_{3}^{P}=K_{3}^{E}=1.05, K_{4}^{P}=K_{4}^{E}=0.95$, $K_{5}^{P}=K_{5}^{E}=1.2, K_{6}^{P}=K_{6}^{E}=0.9, K_{7}^{P}=K_{7}^{E}=1.25$, $K_{1}^{Q}=1.1, K_{2}^{Q}=1.05, K_{3}^{Q}=1.4, K_{4}^{Q}=1.35, K_{5}^{Q}=0.95$, $K_{6}^{Q}=1.23, K_{7}^{Q}=1.08, \alpha=0.5$.

\section{A. Case 1: Controller Performance}

In this case, the performance of the proposed cooperative controller is tested in an islanded microgrid where all the batteries are working in discharging mode to satisfy the demands of loads. The communication network between each BESS is presented in Fig. 4(a). The system reaches a steady state from the beginning due to the operation of primary droop control, and the proposed finite-time consensus control is activated at $t=30 \mathrm{~s}$. In order to verify the robust performance of the proposed controller, a $0.5+0.5 j \mathrm{p}$.u load is removed at $t=80$ $\mathrm{s}$, and an additional $1+1 j$ p.u load is attached to the system at $t=120 \mathrm{~s}$. The simulation results are shown in Fig. 5 .

As can be seen both voltage amplitude and frequency of each BESS deviate from their reference values due to the droop characteristic of the primary control in the first 30 seconds. When our distributed finite-time control is activated at

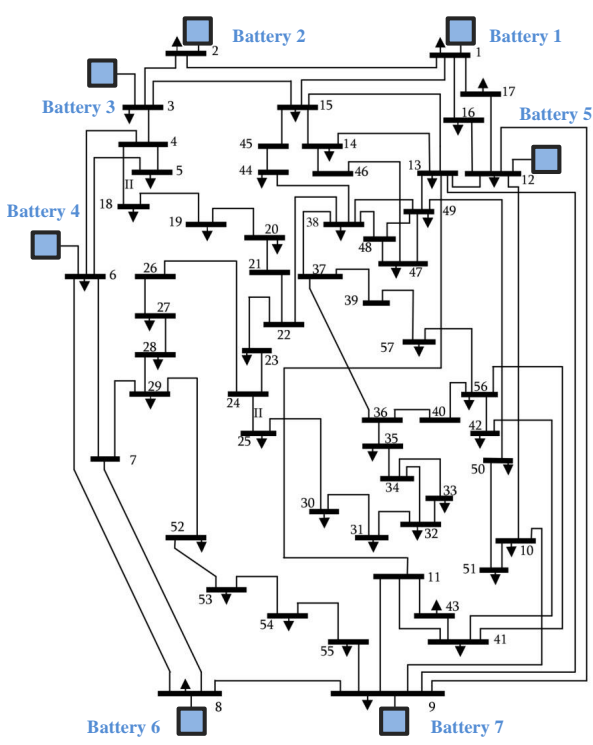

Fig. 3. Test system based on the IEEE 57-bus system

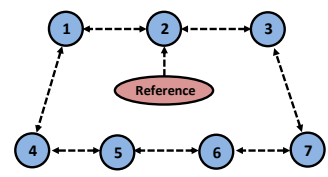

(a)

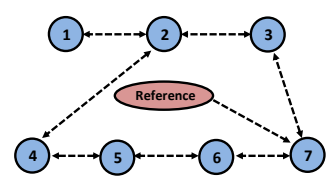

(c)

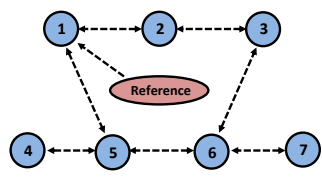

(b)

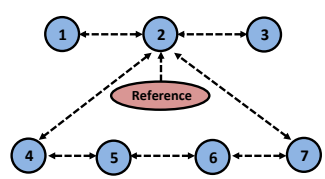

(d)
Fig. 4. Communication networks of the BESSs

$t=30 \mathrm{~s}$, the derivations of voltage and frequency are quickly eliminated. Meanwhile, energy levels and battery powers of BESSs rapidly achieve consensus following the application of the proposed controller, where the energy levels reflect the remaining energy of each battery and the battery powers represent the output active powers provided by the BESSs to satisfy the demands of loads in the microgrid. It should be noted that the energy levels of BESSs are regulated by the injected control inputs through the battery converters such that energy level balancing and active power sharing can be achieved independently. In the following, the performance of the islanded microgrid with respect to additional load is investigated. It can be seen that the steady state of both voltage and frequency remain at their reference values regardless of load changes in the system. The synchronization of energy level and active/reactive power sharing can also be guaranteed. Obviously, the proposed finite-time controller shows good tracking and robust performance during this stage.

\section{B. Case 2: Grid-Connected Mode with Time-Varying Commu- nication Topologies}

In this case study, the performance of the proposed method is validated in a different operating mode and time-varying 

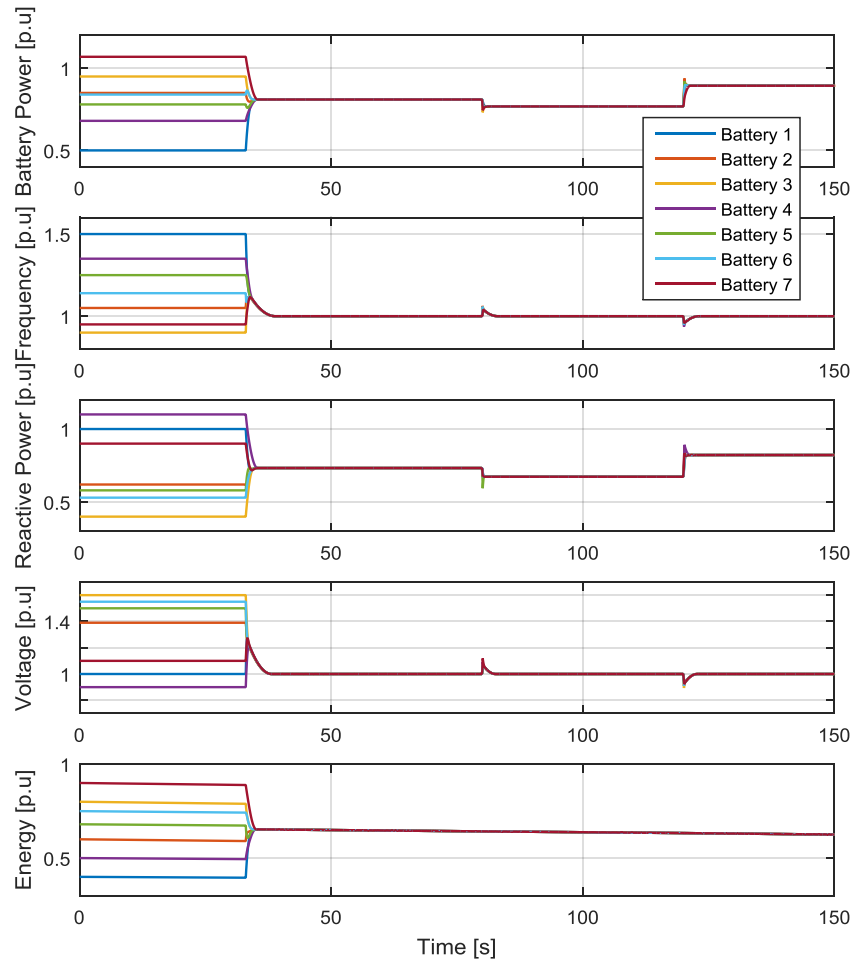

Fig. 5. Simulation results of modified IEEE 57-bus system in Case 1.

communication topologies. All the BESSs are operating in charging mode (grid-connected mode). When the proposed distributed finite-time control is activated after $30 \mathrm{~s}$, the communication topologies in Fig. 4 are switched every 10 seconds according to the sequence: $(a) \rightarrow(b) \rightarrow(c) \rightarrow(d) \rightarrow(a)$. A load increase to the amount of $0.5+0.5 j$ p.u occurs after $75 \mathrm{~s}$.

Simulation results and control inputs of the energy levels and battery powers are illustrated in Fig. 6 and Fig. 7 respectively. It is seen that the proposed design is able to operate in both grid-connected and islanded modes, and is robust to switching topologies. Note that when some energy storage devices suffer from communication failure, the remaining BESSs in the communication networks can still achieve consensus if they are connected to the communication network by choosing an appropriate communication topology, which guarantees the reliability and stability of the whole microgrid.

\section{Case 3: Effect of Consensus Parameters}

The parameters of the proposed controllers have a direct impact on the dynamic response of the energy storage systems. From Section III, the convergence time can be set by tuning the consensus gains $c_{E}, c_{P}, c_{Q}, c_{V}$, and $c_{\omega}$, while the upper bound of finite settling time can be adjusted by selecting a suitable $\alpha$ between 0 and 1 . The communication graph in this case is shown in Fig. 4(c).

First, the effect of consensus gains is investigated, where $c_{V}$ and $c_{\omega}$ in voltage and frequency restoration control are considered and a similar analysis can be applied to other three consensus gains. With $\alpha$ chosen by 0.5 as in Case 1, different $c_{V}$ and $c_{\omega}$ are applied to the BESSs in modified IEEE-57 bus

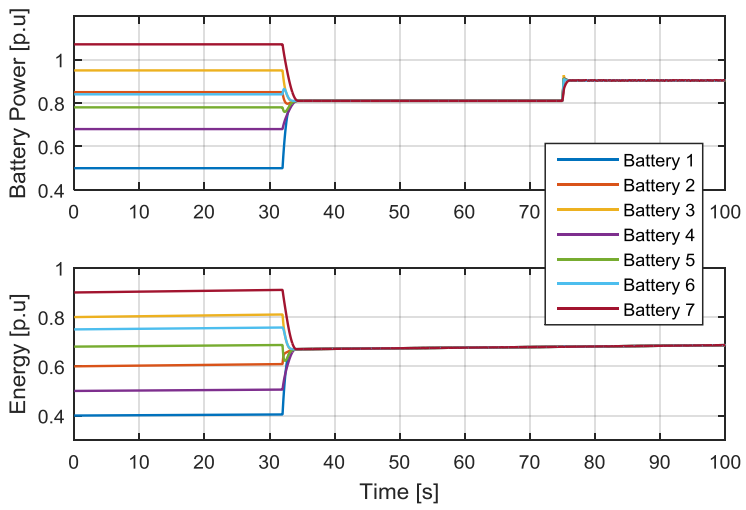

Fig. 6. Simulation results of modified IEEE 57-bus system in Case 2.

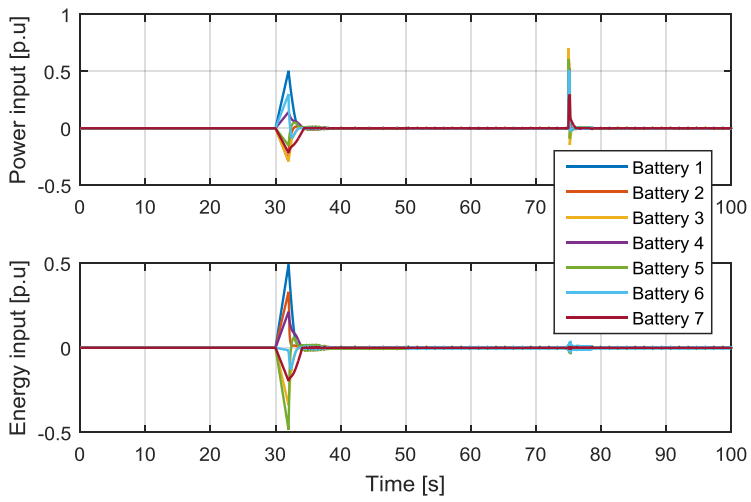

Fig. 7. Control inputs in Case 2.

system. It can be seen from the simulation results in Fig. 8 that the convergence speed of the system will increase by selecting a higher consensus gain. However, these consensus gains can not be arbitrarily large when considering the physical limitations of the control inputs to BESSs.

Next, the system performances of battery power and reactive power in BESSs with different $\alpha$ are analyzed. Consensus gains $c_{P}$ and $c_{Q}$ are chosen by 0.45 and 0.5 , respectively. As shown in Fig. 9, with a smaller $\alpha$, the responses of the BESSs reach consensus in a shorter time. Meanwhile, the performance of the system becomes more aggressive when decreasing the value of $\alpha$. As a result, there is a trade-off between the system performance and convergence speed when choosing a suitable $\alpha$ to meet the requirement of the microgrids.

\section{Case 4: Comparing the Proposed Method with the Ap- proach in [13]}

In this section, we compare our proposed distributed finitetime controller with the approach presented in [13], which is one of the most representative distributed control methods of energy storage systems. Note that the consensus protocol design in [13] requires global information from the communication graph structure. Thus, when the size of the microgrids increases or the communication graph topology changes, degradation of controller performance will be the result. Alternatively, the proposed method in this paper is 

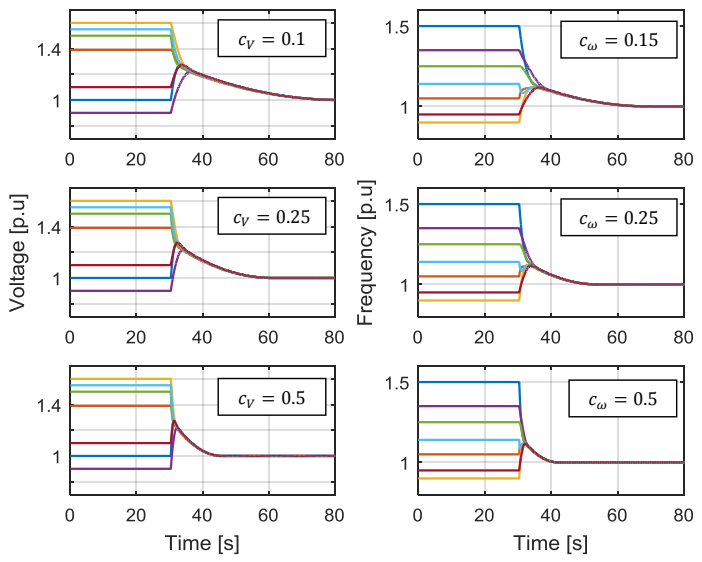

Fig. 8. Simulation results for different consensus gains in Case 3 .
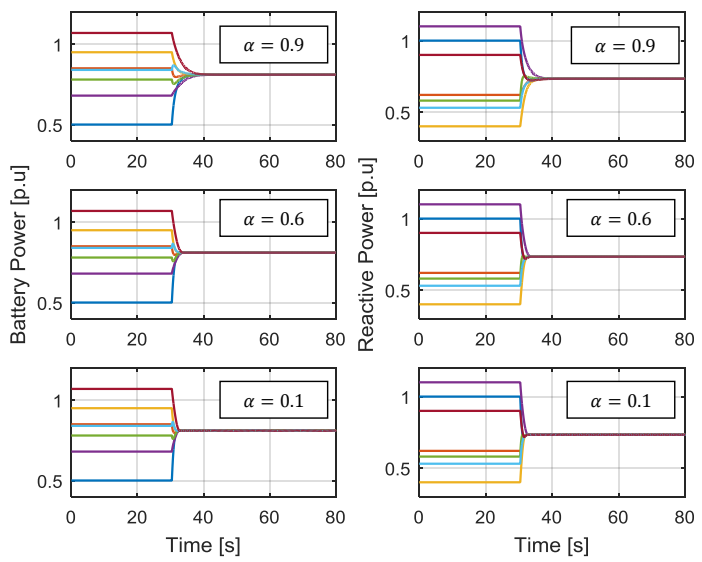

Fig. 9. Simulation results for different $\alpha$ in Case 3 .

fully distributed, which is suitable for large-scale networked microgrid systems. Furthermore, the control protocol in [13] is an asymptotic controller which can only guarantee global asymptotic stability of the energy storage devices, whereas our proposed method is able to achieve consensus within a finite time period.

A comparison with the conventional method proposed in [13] is made by applying it to the same droop-based microgrid as in Case 1. The control parameters are set the same in both our proposed method and the conventional controller in [13]. The simulation results are shown in Fig. 10, where only responses of battery power, voltage magnitude and frequency in BESS 1 are depicted for simplicity. It can be seen that our proposed controller leads to a faster convergence speed and shows more accurate robust performance for controller activation and load changes.

\section{CONCLUSION}

In this paper, a fully distributed finite-time control architecture for heterogeneous battery energy storage system in droop-controlled microgrids is presented. The proposed distributed method is shown to achieve energy level balancing, active/reactive power sharing, and voltage/frequency synchronization of energy storage devices in a finite time period. Each battery storage system only communicates with its neighbors through a sparse communication topology, thus providing a robust hierarchical control framework that appropriately operates in the presence of time-varying and unreliable communication networks. This yields better extensibility and flexibility when compared with centralized control approaches. The effectiveness of the proposed strategy is validated by simulations, where the robustness of the modified IEEE 57-bus power system is investigated under different disturbances such as load changes and time-varying communication topologies. Future works will investigate optimal resource management in a microgrid based on a multi-agent system framework, and factors such as supply-demand balance and energy costs will be taken into consideration in the optimization design.

\section{REFERENCES}

[1] A. Bidram, F. L. Lewis, and A. Davoudi, "Distributed control systems for small-scale power networks: Using multiagent cooperative control theory," IEEE Control Systems, vol. 34, no. 6, pp. 56-77, 2014

[2] F. Guo, C. Wen, J. Mao, and Y.-D. Song, "Distributed secondary voltage and frequency restoration control of droop-controlled inverter-based microgrids," IEEE Transactions on Industrial Electronics, vol. 62, no. 7, pp. 4355-4364, 2015.

[3] W. Tushar, B. Chai, C. Yuen, S. Huang, D. B. Smith, H. V. Poor, and Z. Yang, "Energy storage sharing in smart grid: A modified auctionbased approach," IEEE Transactions on Smart Grid, vol. 7, no. 3, pp. 1462-1475, 2016.

[4] C. A. Hill, M. C. Such, D. Chen, J. Gonzalez, and W. M. Grady, "Battery energy storage for enabling integration of distributed solar power generation," IEEE Transactions on Smart Grid, vol. 3, no. 2, pp. 850-857, 2012.

[5] W. Huang and J. A. A. Qahouq, "Energy sharing control scheme for state-of-charge balancing of distributed battery energy storage system," IEEE Transactions on Industrial Electronics, vol. 62, no. 5, pp. 2764 2776, 2015.

[6] S. Zuo, A. Davoudi, Y. Song, and F. L. Lewis, "Distributed finitetime voltage and frequency restoration in islanded ac microgrids," IEEE Transactions on Industrial Electronics, vol. 63, no. 10, pp. 5988-5997, 2016.

[7] A. Bidram and A. Davoudi, "Hierarchical structure of microgrids control system," IEEE Transactions on Smart Grid, vol. 3, no. 4, pp. 1963-1976, 2012.

[8] J. M. Guerrero, J. C. Vasquez, J. Matas, L. G. De Vicuña, and M. Castilla, "Hierarchical control of droop-controlled ac and dc microgridsa general approach toward standardization," IEEE Transactions on Industrial Electronics, vol. 58, no. 1, pp. 158-172, 2011.

[9] J. Wang, A. Lanzon, and I. R. Petersen, "Robust cooperative control of multiple heterogeneous negative-imaginary systems," Automatica, vol. 61, pp. 64-72, 2015.

[10] N. M. Dehkordi, N. Sadati, and M. Hamzeh, "Distributed robust finitetime secondary voltage and frequency control of islanded microgrids," IEEE Transactions on Power Systems, vol. 32, no. 5, pp. 3648-3659, 2017.

[11] J. Wang, A. Lanzon, and I. R. Petersen, "Robust output feedback consensus for networked negative-imaginary systems," IEEE Transactions on Automatic Control, vol. 60, no. 9, pp. 2547-2552, 2015.

[12] D. Bian, M. Kuzlu, M. Pipattanasomporn, and S. Rahman, "Analysis of communication schemes for Advanced Metering Infrastructure (AMI)," in Proceedings of IEEE PES General Meeting | Conference \& Exposition. IEEE, 2014, pp. 1-5.

[13] T. Morstyn, B. Hredzak, and V. G. Agelidis, "Distributed cooperative control of microgrid storage," IEEE Transactions on Power Systems, vol. 30, no. 5, pp. 2780-2789, 2015.

[14] T. Morstyn, M. Momayyezan, B. Hredzak, and V. G. Agelidis, "Distributed control for state-of-charge balancing between the modules of a reconfigurable battery energy storage system," IEEE Transactions on Power Electronics, vol. 31, no. 11, pp. 7986-7995, 2016.

[15] T. Morstyn, B. Hredzak, and V. G. Agelidis, "Control strategies for microgrids with distributed energy storage systems: An overview," IEEE Transactions on Smart Grid, 2016. 


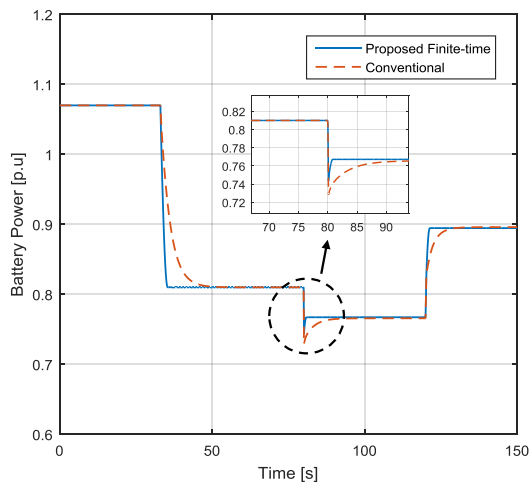

(a)

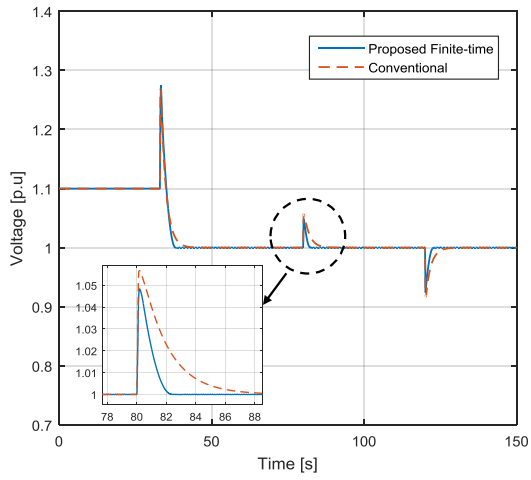

(b)

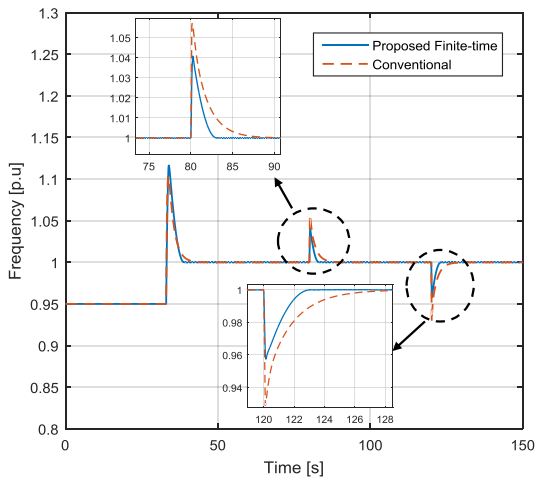

(c)

Fig. 10. Performance comparison of the proposed finite-time method and the asymptotic controller in [13]: (a) battery power. (b) voltage. (c) frequency.

[16] Y. Xu, W. Zhang, G. Hug, S. Kar, and Z. Li, "Cooperative control of distributed energy storage systems in a microgrid," IEEE Transactions on Smart Grid, vol. 6, no. 1, pp. 238-248, 2015.

[17] T. Morstyn, B. Hredzak, and V. G. Agelidis, "Cooperative multi-agent control of heterogeneous storage devices distributed in a DC microgrid," IEEE Transactions on Power Systems, vol. 31, no. 4, pp. 2974-2986, 2016.

[18] J. Khazaei and D. H. Nguyen, "Multi-agent consensus design for heterogeneous energy storage devices with droop control in smart grids," IEEE Transactions on Smart Grid, (In press), DOI:10.1109/TSG.2017.2765241.

[19] Z. Miao, L. Xu, V. R. Disfani, and L. Fan, "An SOC-based battery management system for microgrids," IEEE Transactions on Smart Grid, vol. 5, no. 2, pp. 966-973, 2014.

[20] J. Khazaei and Z. Miao, "Consensus control for energy storage systems," IEEE Transactions on Smart Grid, vol. 9, no. 4, pp. 3009-3017, 2018.

[21] C. Huang, S. Weng, D. Yue, S. Deng, J. Xie, and H. Ge, "Distributed cooperative control of energy storage units in microgrid based on multiagent consensus method," Electric Power Systems Research, vol. 147, pp. 213-223, 2017.

[22] D. H. Nguyen and J. Khazaei, "Multi-agent time-delayed fast consensus design for distributed battery energy storage systems," IEEE Transactions on Sustainable Energy, vol. 9, no. 3, pp. 1397-1406, 2018.

[23] X. Liu, J. Lam, W. Yu, and G. Chen, "Finite-time consensus of multiagent systems with a switching protocol," IEEE Transactions on Neural Networks and Learning Systems, vol. 27, no. 4, pp. 853-862, 2016.

[24] G. H. Hardy, J. E. Littlewood, and G. Pólya, Inequalities. Cambridge university press, 1952.

[25] H. Zhang, F. L. Lewis, and Z. Qu, "Lyapunov, adaptive, and optimal design techniques for cooperative systems on directed communication graphs," IEEE Transactions on Industrial Electronics, vol. 59, no. 7, pp. 3026-3041, 2012.

[26] M. Forti, M. Grazzini, P. Nistri, and L. Pancioni, "Generalized lyapunov approach for convergence of neural networks with discontinuous or nonlipschitz activations," Physica D: Nonlinear Phenomena, vol. 214, no. 1, pp. 88-99, 2006.

[27] A. Lanzon and I. R. Petersen, "Stability robustness of a feedback interconnection of systems with negative imaginary frequency response," IEEE Transactions on Automatic Control, vol. 53, no. 4, pp. 1042-1046, 2008.

[28] A. Lanzon and H.-J. Chen, "Feedback stability of negative imaginary systems," IEEE Transactions on Automatic Control, vol. 62, no. 11, pp. 5620-5633, 2017.

[29] X. Li, D. Hui, and X. Lai, "Battery energy storage station (BESS)-based smoothing control of photovoltaic (PV) and wind power generation fluctuations," IEEE Transactions on Sustainable Energy, vol. 4, no. 2, pp. 464-473, 2013.

[30] G. He, Q. Chen, C. Kang, Q. Xia, and K. Poolla, "Cooperation of wind power and battery storage to provide frequency regulation in power markets," IEEE Transactions on Power Systems, vol. 32, no. 5, pp. 35593568, 2017.

[31] S. Teleke, M. E. Baran, A. Q. Huang, S. Bhattacharya, and L. Anderson, "Control strategies for battery energy storage for wind farm dispatching,"
IEEE Transactions on Energy Conversion, vol. 24, no. 3, pp. 725-732, 2009.

[32] A. Bidram, A. Davoudi, and F. L. Lewis, "Finite-time frequency synchronization in microgrids," in Proceedings of IEEE Energy Conversion Congress and Exposition (ECCE), 2014, pp. 2648-2654.

[33] R. Christie and I. Dabbagchi, "IEEE 57-bus system," 1993. [Online]. Available: http://item.bettergrids.org/handle/1001/225

[34] M. Chen and G. A. Rincon-Mora, "Accurate electrical battery model capable of predicting runtime and IV performance," IEEE Transactions on Energy Conversion, vol. 21, no. 2, pp. 504-511, 2006.

[35] L. Harnefors, M. Bongiorno, and S. Lundberg, "Input-admittance calculation and shaping for controlled voltage-source converters," IEEE Transactions on Industrial Electronics, vol. 54, no. 6, pp. 3323-3334, 2007.

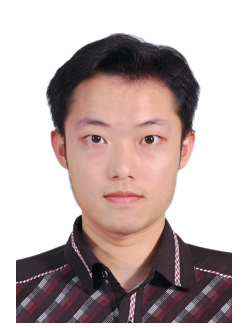

Junyan Hu received the B.Eng degree in Automation from Hefei University of Technology in 2015 and M.Sc degree in Advanced Control and System Engineering from the University of Manchester in 2016, where he is currently working toward the Ph.D degree in Electrical and Electronic Engineering. His research interests include distributed cooperative control of multi-agent dynamical systems and control application for microgrids and energy storage systems.

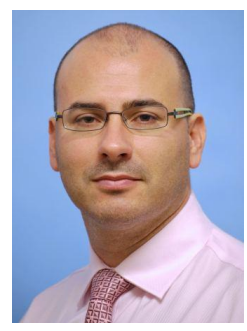

Alexander Lanzon received his Ph.D. degree in Control Engineering and his M.Phil. degree in Robot Control from the University of Cambridge in 2000 and 1997 respectively and received his B.Eng.(Hons). degree in Electrical and Electronic Engineering from the University of Malta in 1995. Alexander held academic positions at Georgia Institute of Technology and the Australian National University, and industrial posts at ST-Microelectronics (Malta) Ltd., Yaskawa Denki (Tokyo) Ltd. and National ICT Australia Ltd., before joining the University of Manchester in 2006 where he now holds the Chair in Control Engineering. Alexander is a Fellow of the Institute of Mathematics and its Applications, the Institute of Measurement and Control and the Institution of Engineering and Technology. He is also an Associate Editor of the IEEE Transactions on Automatic Control and has served as a Subject Editor of the International Journal of Robust and Nonlinear Control. His research interests include the fundamentals of feedback control theory, negative imaginary systems theory, $H_{\infty}$ control theory, robust linear and nonlinear control systems, and applying robust control theory to innovative mechatronic, robotic and drone applications. 